\title{
LA ECOLOGIA EN LOS PROYECTOS EDUCATIVOS INSTITUCIONALES ACORDE A LAS NUEVAS NORMAS
}

Por ALVARO AVENDAÑO R. Profesor Universidad Surcolombiana

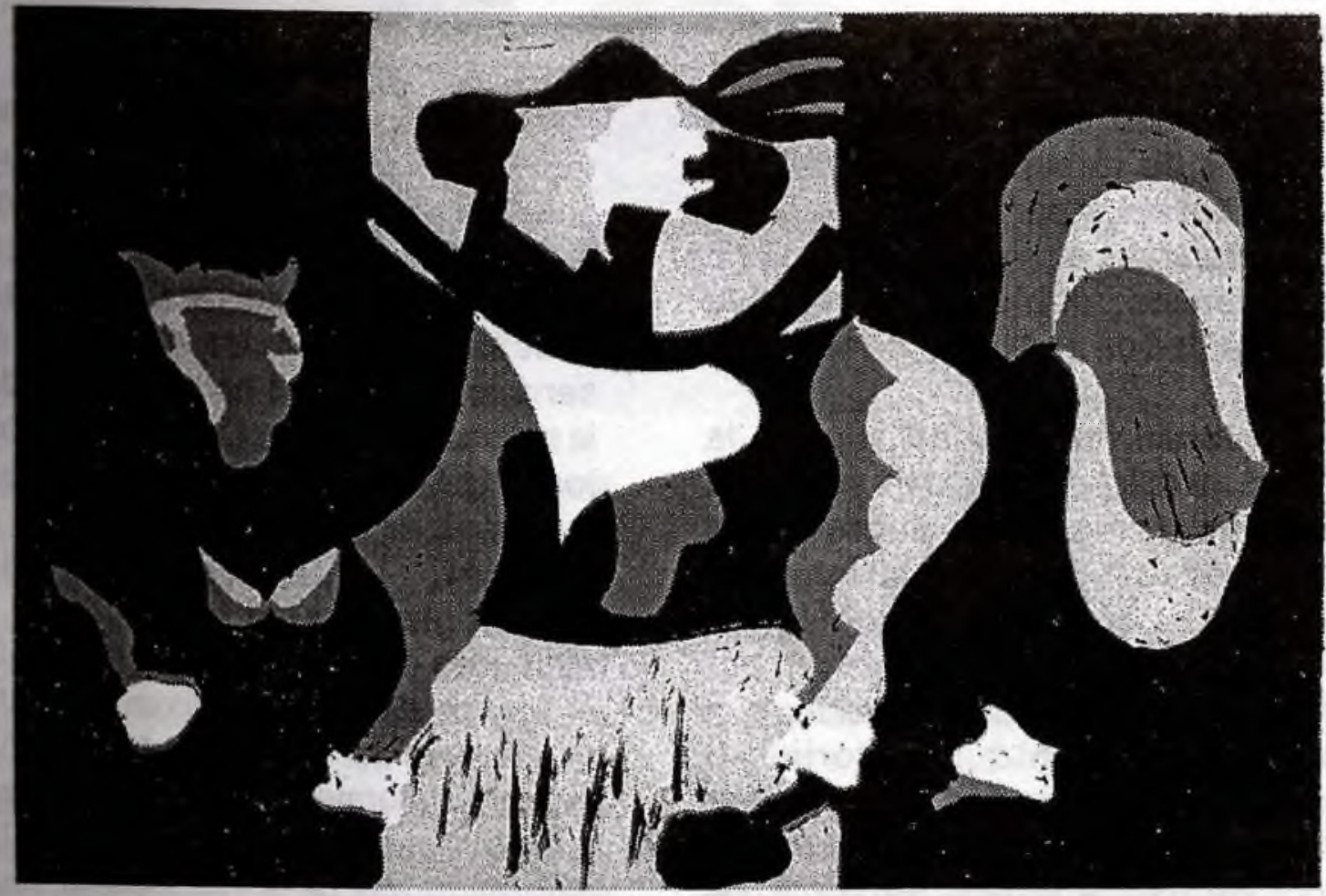

\section{MARCO CONCEPTUAL}

Para que el proceso de modernización no se quede en lo instrumental, el desarrollo humano que se derive debe enmarcarse dentro de la perspectiva de maduración de la sociedad civil a través de su participación en todos los procesos contemplados en la constitución de 1991 y sus leyes reglamentarias, en particular la ley general de educación y la de participación ciudadana.

Se propone que la participación ciudadana en las instituciones educativas se organice entorno al problema ambiental, pues para abordarlo es requisito fundamental la interdisciplinariedad. Esta característica lo convierte en un punto 
estratégico para una educación cuyo currículo se desarrolle en torno a problemas, más que a asignaturas o discplinas en particular.

La interdisciplinariedad es necesaria en cuanto que la crisis del medio ambiente ha puesto en claro la ambivalencia no sólo de la organización social, sino también de las formaciones cientificas.

Una de las críticas que viene planteando el amblentalismo al desarrollo de la ciencia es la incapacidad de la misma para el análisis de sistemascomplejos. La dificultad surge por la incapacidad de las diferentes disciplinas del diálogo académico, debido a la relativa insularidad de cada una de ellas.

La interdisciplinariedad supone la apertura epistemológica de cada una de las ramas del saber para fecundación del diálogo interdisciplinario.

La gestión ambiental se relaciona con el ordenamiento territorial o sectorización de las actividades humanas, con la planeación urbana, por ejemplo la fijación de zonas de descanso y recreo y la organización de sus servicios para mantener ambiente sano y agradable para la comunidad (código del régimen municipal Art. 34), la ejecución de planes de desarrollo urbano y la constitución de zonas de reservas para futuras extensiones de las ciudades, o para la protección del sistema ecológico, son motivos de utilidad pública o interés social (Código del Régimen Municipal Art. 43).

La gestión ambiental se relaciona con la cultura pues la forma en que los individuos intentan resolver los problemas está intrínsecamente relacionada con los valores y las metas de la sociedad, con los instrumentos y con las instituciones ${ }^{1}$.

Por ejemplo el hombre precolombino se relacionó con su entorno con un gran respeto y admiración sagrada por la naturaleza externa: su técnica formaba parte de las mismas enseñanzas naturales y estaba al servicio no sólo del hombre, sino de la naturaleza exterior. Cuando llegaron los españoles y en general los europeos, el paradigma comenzó a cambiar, la técnica llamada tecnología, era el gesto, que a mediados del siglo XVII y XVIII tenía el sentido de utilidad máxima puesta al servicio del hombre. La acción sobre la naturaleza exterior comenzó a sobrepasar los límites de la diferencia, no para reafirmarla,sino para desaparecerla, y como consecuencia, toda acción se centró de una manera $u$ otra en el hombre. El nuevo paradigma era el hombre, el hombre medida de todas las cosas, punto de partida y punto de llegada

1. ROGOLF, B. Aprendices del pensamiento. El desarrollo en el contexto social. Barcelona. Buenos Aires. 1993 pp.71-93. Citado por CINDE en Cognición y Desarrollo Humano. Serie Postgrado. Neiva. 
de todas las acciones creadoras de la cultura $^{2}$.

Y ese gran respeto y admiración casi sagrado por la naturaleza externa es donde debe codificarse la tecnología y volver a decodificarse de acuerdo a los paradigmas del hombre precolombino donde el hombre era punto de partida y de llegada desde allí debe ser abordada la cotidianidad de las comunidades.

La gestión $\mathrm{a} m \mathrm{~b}$ i e n t a l conduce a la autonomía de la comunidad se genera a partir de p rocesos culturales de conocimiento $y$ solución de sus problemas, mediante la investigación participativa en sus diferentes modalidades, asumiendo un saber científico y técnico que propicie un diálogo, permitiendo así una educación democrática y autónoma, que sea un proceso de crecimiento en las potencialidades de la población para resolver sus problemas y desarrollar actitudes críticas. En ese espacio cultural hay necesidad de articular el dominio de la naturaleza con la organización racional de la interacción humana ${ }^{3}$.
En gestión ambiental no existen paradigmas y el paradigma de la tecnología se refiere a patrones importados y que son en general, demostraciones ineficaces 0 sumamente costosos en relación a los recursos disponibles ${ }^{4}$.

La gestión ambiental se relaciona con la ética y el derecho: La importancia de la normatividad ética y jurídica para orientar el comportamiento frente al medio es de una importancia capital. Muchos de los problemas ambientales modernos se pueden deber a la conciencia jurídica instalada en Occidente desde el Derecho Romano.

La gestión ambiental devela el papel de la ciencia. La ciencia es la expresión simbólica por excelencia de la mentalidad moderna. Sus paradigmas han sido definidos por la racionalidad occidental. La perspectiva está definiendo algunos límites de la ciencia moderna, al menos como instrumento adaptativo. Una aproximación teórica a la ciudad no puede hacerse sin intentar una

2. NOGUERA, Patricia. La crisis del medio ambiente en la modernidad: urgencia de una nueva eticidad. Memorias del primer seminario latinoamericano sobre habitat urbano y medio ambiente. ICFES.Universidad Nacional,seccional Manizales. 1991. p. 56.

3. NAVARRO, Wisberto. GEAUR. Marco teórico. Mayo de 1994.

4. NEIRA, Eduardo Alva. Habitat humano y educación ambiental. Memorias del primer seminario latinoamericano sobre habitat urbano y medio ambiente. ICFES.Universidad Nacional de Colombia, seccional Manizales,1991. p. 44. 


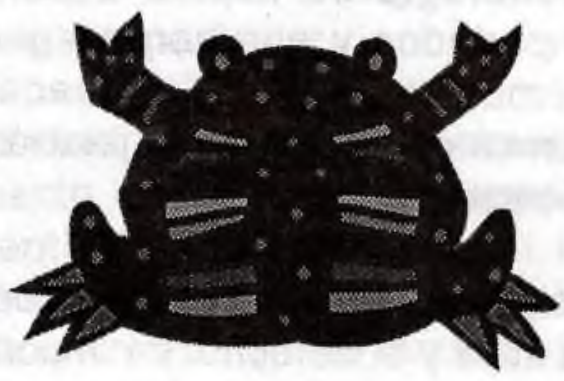

aproximación a como la ciencia ha organizado las redes urbanas.

La gestión ambiental exige una utilización estética de los materiales locales. No se han realizado todavía análisis ambientales del palsajismo colombiano, tan importante para estudiar las condiciones ambientales de épocas anteriores.

La expresión literaria es una fuente insospechada de investigación tanto urbana como ambiental.

En el tercer milenio se impondrá el mercado de los bienes sociales y los de conocimiento ${ }^{5}$.

La gestión ambiental requiere de un desarrollo amplio de ese mercado informático para que las comunidades tengan acceso a la información necesaria, en la cantidad necesaria y al nivel necesario para hacer su gestión ambiental. La información debe definirse como espacio público, de uso de la comunidad.

La educación ambiental, en la medida en que ofrece los conocimientos para la gestsión ambiental, no debe verse separada de educación para la democracia, pues ofrece un amplio campo para explorar la posibilidad de que los problemas ambientales locales se discutan dentro de un proceso de formación para democracia y de la convivencia pacífica.

La democracia que se propone desarrollar es de tipo participativo a través de los siguientes mecanismos: Referendum (citado por el presidente de la república), plebiscito, asamblea nacional constituyente, revocatoria del mandato y voto programático.

\section{LEGISLACION}

Existe más de un centenar de decretos, leyes, acuerdos y resoluciones normativas sobre normas y disposiciones previstas por la ley en participación, mecanismos de participación, normas ambientales, mecanismos de defensa. En la constitución existen 17 artículos sobre normas, 5 sobre participación, 4 sobre planificación y 6 sobre mecanismos de defensa del medio ambiente.

5. GRANE, Herwitte d. EL NUEVO MERCADO DE INTERCAMBIO SOCIAL ABLEX PUBLISHING CORPORATION, New Jersey. 1980. UNIANDES. Magister en dirección universitaria. p.9 


\subsection{Principales medidas de descentralización}

Existe un proceso de descentralización administrativa, política y fiscal de la planificación que viene desarrollándose en el país desde hace varias décadas y con el cual se pretende crear las bases para la participación regional y local en la gestión del desarrollo social, económico y ambiental.

Las medidas que reflejan este proceso son: las de planificación regional: Ley 76 de 1985 por la cual se crean las regiones de planificación. Sobre descentralización fiscal: Ley 14 de 1983: Normas sobre catastro, impuesto predial e impuesto de renta y complementarios; impuesto de industria y comercio; impuesto de circulación y tránsito y timbre; impuesto al consumo de licores y de cigarrillos; impuesto a la gasolina, sobre descentralización política y administrativa: Elección de alcaldes (acto número 1 de 1986) y la ley II de 1986 por la cual se dictó el estatuto básico de la administración municipal y se ordena la participación de la comunidad en el manejo de los asuntos locales. Esta ley se reemplaź por la ley 136 de $1994^{\circ}$.
Sin embargo, es bueno acotar que el neo-estructuralismo (política samperista) es estatista, centralizador $y$, en fin de cuentas, contrario a la libertad, lo que pone en peligro la descentralización sin reivindicar el esquema regional basado en las ventajas comparativas y competitivas ${ }^{7}$.

\subsection{La participación cludadana}

\section{La participación ciudadana está contemplada en el Art. 79 de la Constitución Nacional.}

La participación ciudadana, en cuanto a gestión ambiental, se garantiza mediante la ley 99 de diciembre de 1993 con la que se crea la estructura del Ministerio del Medio Ambiente. En la estructura se contempla la subdirección de participación ciudadana y relaciones con la comunidad y la subdirección de evaluación, seguimiento y asesoría regional. La participación ciudadana se da a través de veedurías ante la Procuraduría delegada para asuntos ambientales y acciones de cumplimiento. Además el Ministerio del Medio Ambiente cuenta con el apoyo de instituciones de

6. ZAPATA, Roberto. Descentralización y autonomía regional. Modulo 4. Maestría CINDE. Universidad Surcolombiana Neiva, Agosto de 1994. p. 8.

7. ZAPATA, Roberto. Descentralización y autonomía regional. módulo 4. Maestría CINDE. Universidad Surcolombiana, neiva, agosto de 1994. p. 9. 
investigación, corporaciones de régimen especial y universidades los cuales son otros tantos caminos de injerencia de la comunidad en las decisiones en cuanto a lo ambiental ${ }^{8}$.

\section{GESTION AMBIENTAL}

En el plan decenal de educación ${ }^{9}$ se señala como la séptima estrategia: la promoción de la cultura y ampliación del horizonte educativo.

La gestión ambiental deberá identificar los instrumentos políticos, económicos, tecnológicos, sociales y/o normativos que permitan orientar tendencias regionales con criterio de desarrollo sostenible pues ella debe llevar al fortalecimiento del sector agroindustrial e industrial, fundamentado en el desarrollo de la investigación científica y tecnológica sin descuidar un adecuado manejo del sistema agroalimentario regional que fortalezca y racionalice la distribución y el consumo interno de importancia para el potencial ecosistémico de la región.

La gestión ambiental aumentará la participación comunitaria fundamentada en la educación ambiental para así poder propender por la conservación, recuperación y mejoramiento físico-espacial de las estructuras urbanas y rurales, fundamentales en la búsqueda de una mejor calidad de vida.
La gestión ambiental es necesario articularla con el sistema nacional para la gestión nacional SINA y con las actividades desarrolladas por el Ministerio del Medio Ambiente (Ley 99 del 22 de diciembre de 1993).

Los instrumentos legales con que cuenta la comunidad son:

Las acciones ambientales pueden darse; a través de las juntas administradoras locales para comunas y corregimientos (Art. 318 C.N. Art. 157 a 160 del Código de Régimen Municipal).

Las personas tienen el derecho a obtener información de las autoridades sobre sus planes y actos y el deber de éstas a proporcionarla (Decreto 01 de $1984 \mathrm{C}$. Contencioso Administrativo, Art. 17 al 20) además del derecho de petición en interés general o particular, según los alcances del asunto (Art. 5 al 8 y 9 al 16 del Código Contencioso Administrativo).

\section{LOS P.E.I. Y LOS PROYECTOS AMBIENTALES}

En la Ley General de Educación, en la parte curricular del P.E.I., se contempla el área de educación ambiental y la educación para la democracia. También en la Ley 99 de diciembre de 1993 se contempla la prestación del servicio ambiental

8. GEAUR. USCO, 1994.

9. Ministerio de Educación Nacional. "Educación para la democracia, el desarrollo, la equidad y la convivencia". Colección Plan Decenal de Educación, 1996. 


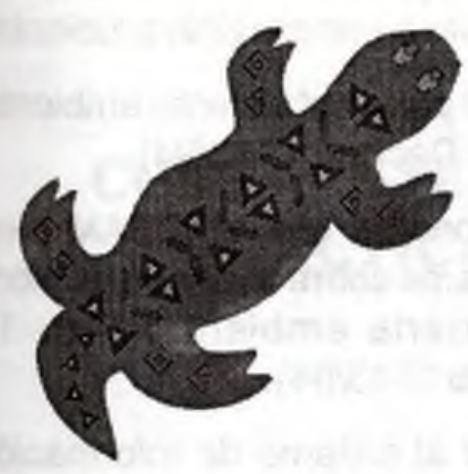

obligatorio. La educación para la democracia debe ser entendida como una estrategia de formación en los procesos democráticos.

En desarrollo de la Ley General, el Art. 10. del Decreto $1743 / 94$ ordena a todas las instituciones educativas a incluir dentro de sus PEIS proyectos ambientales en el marco de diagnósticos ambientales, locales, regionales y/o nacionales con miras a coadyuvar a la solución de problemas ambientales específicos.

Por otro lado la Ley permite que el servicio social obligatorio sirva de apoyo a los proyectos ambientales y la formación, consolidación de grupos ecológicos o participando en actividades comunitarias de educación ecológica o ambiental (Art. 7 Decreto 1743/94). A esto hay que adicionar que según Art. 57 Decreto 1860 del 3 de agosto de 1994 se deben agregar 10 horas por semana para actividades lúdicas, culturales, deportivas y sociales orientadas por pautas curriculares según se establezca en el P.E.I., según el interés del estudiante.

Es posible ligar este tiempo a las prácticas extraescolares al ampliarse el concepto que se tenía de ellas: EL
ARTICULO 204 de la Ley 115 de 1994 nos define una práctica extraescolar como parte del proceso educativo consistente en la utilización del tiempo libre, en recreación y otras actividades $y$ en propiciar formas asociativas (como grupos ecológicos) para que los educandos complementen la educación ofrecida en la familia y en los establecimientos educativos. Según el Decreto 1860, uno de los contenidos de las prácticas está constituido por las acciones pedagógicas relacionadas con la educación para el ejercicio de la democracia, para la educación sexual, para el uso del tiempo libre, para el aprovechamiento y conservación del medio ambiente $y$, en general, para los valores humanos.

También existe la posibilidad, para los que así lo deseen, de prestar el servicio militar como servicio ambiental (Art. 8 d. y Art. 102 de la Ley 99 de 1993).

Existen recursos para actividades extraescolares: pues la Ley en su Art. 13 de la Ley 115 de 1994 establece financiación con cargo a la participación en los ingresos corrientes de la Nación (destinados por la Ley para tales áreas de inversión social) para los programas que hacen referencia al aprovechamiento del tiempo libre los cualesl serán presentados a las secretarías de Educación. Las actividades pueden conformar proyectos que deben remitirse al Fondo Nacional Ambiental (FONAM) para su financiamiento (Art. 18 Decreto 1743/94)

También se puede contar con el recurso humano de las Facultades de Educación para desarrollo de 
programas de capacitación de los educadores en pregrado y postgrado (Art. 5 Decreto 1743/94), para facilitar el flujo de información sobre experiencias e investigaciones sobre educación ambiental entre las instituciones y el MEN y el Ministerio del Medio Ambiente (Art. 4 Decreto 1743/94) y para la evaluación del proyecto ambiental escolar (Art. 6 Decreto 1743/94) en coordinación con el MEN, Ministerio del Medio Ambiente, CAM, y directivas de las instituciones.

En el proyecto ambiental se deben incluir acciones de:

- Coordinación de acciones entre las instituciones educativas y las universidades $u$ otros organismos públicos y privados ubicados en la localidad o región (Art. 3 decreto 1743/94).

- Asesorías posibles de la secretaría de educación para la coordinación control de ejecución de los proyectos ambientales escolares (Art. 4 Decreto 1743/94)

- Coordinación con el MEN,Ministerio del Medio Ambiente, CAM. Secretarías de Educación, Distritos
Militares para que el servicio militar se preste como servicio ambiental.

- Formar parte del sistema ambiental (Art. 9. Decreto 1743/94).

- Relacionarse con el IDEAM para informarse sobre avances técnicos en materia ambiental (Art. 14 Decreto 1743/94).

- Apoyar al sistema de información ambiental (Art. 16 Decreto 1743/94)

- Programas de investigación en sobre el contexto social y cultural regional, ofertas y demandas ambientales de la región, sobre cuales son los conocimientos pertinentes al medio de los educandos para mejorar su calidad de vida.

- Programas de uso de la T.V. regional: Contemplada en el plan decenal ${ }^{10}$ de educación como la segunda estrategia para elevar la calidad de la educación. La nueva Ley de T.V. apoya este tipo de televisión para la gestión y el desarrollo de las comunidades, el canal de T.V. comunal. La T.V. es un gran espacio para sensibilizar a la población sobre los problemas ambientales.

10. Ministerio de Educación Nacional. "Educación para la democracia, el desarrollo la equidad y la convivencia", Colección Plan decenal de Educación. 1996. 